\title{
Heaven - Theaterpädagogisch Vor- und nachbereitet
}

\author{
Bärbel Jogschies / Doris Krohn
}

\begin{abstract}
Zusammenfassung
Das Berliner Maxim Gorki Theater bietet wie viele deutsche Theater für Zuschauergruppen und Schulklassen theaterpädagogische Workshops an, die das Publikum auf den Theaterbesuch vorbereiten und in die Inszenierungen einführen. Diese Workshops enthalten interaktive Spiele und Übungen, die die Absichten der Theatermacher nachempfinden lassen und das Verständnis für die spezielle Theaterarbeit erhöhen. Die Autorinnen glauben, dass sich diese spielerischen Interaktionen für den Fremdsprachenunterricht eignen. Exemplarisch wird im Teil I von der Theaterpädagogin Bärbel Jogschies ein solcher Workshop beschrieben. Im Teil II reflektiert die Deutsch als Fremdsprache-Dozentin Doris Krohn über die Wirkungen, die dieser Workshop und der daran anschließende Theaterbesuch auf die Teilnehmer hatten. Nicht zuletzt ist der Artikel ein Kulturtipp, eine Leseempfehlung und eine Liebeserklärung an das moderne deutsche Theater.
\end{abstract}

1 Teil

Theaterpädagogin Bärbel Jogschies beschreibt einen theaterpädagogischen Einführungsworkshop in die Armin Petras' Inszenierung Heaven (zu Tristan) von Fritz Kater am Maxim Gorki Theater Berlin ${ }^{1}$

\subsection{DAS STÜCK}

Liebestod in Wolfen: Simone liebt Anders. Doch wie einst Tristan seine Heimat Cornwall, verlässt der Architekturstudent Anders Wolfen, die Stadt der gemeinsamen Kindheit. Er bricht auf nach Amerika. Simone bleibt in der ostdeutschen Provinz zurück. Helga liebt ihren Mann, den Psychiater Königsforst, und das schon seit mehr als zwanzig Jahren. Doch auch sie werden Wolfen verlassen. Seit die Filmfabrik geschlossen wurde, gibt es für die Laborantin keine Arbeit mehr. Gerade noch haben sie gespielt, ßterne am

${ }^{1}$ Vgl. in diesem Kontext auch das Interview mit Armin Petras in SCENARIO 2 (2007). 
himmel des anderen“ zu sein. Jetzt wird ihr Neubaublock abgerissen. Zurück bleiben die Reste und die Raben.

„erleben wir uns als in die welt geschleudert oder als teil eines ganzen?“

Wie Marietta Blau, die einst dank der Entwicklung einer speziellen Filmemulsion die sternenförmig auseinander fliegenden Teilchen einer Kernzertrümmerung sichtbar machte, beschreibt Fritz Kater mit "Heaven"die Zertrümmerung einer Lebenslandschaft und zeigt deren Folgen. Kater führt seine Figuren vom Paradies durch das Fegefeuer in die gegenwärtige Hölle des deutschen Ostens.

So kündigte das Maxim Gorki Theater Berlin die Uraufführung des Stückes Heaven (zu Tristan) von Fritz Kater an. Kein leichter Stoff. Sowohl die literarische Vorlage als auch der inszenatorische Zugriff erfordern viel Insiderwissen, sowohl was die Situation in Ostdeutschland als auch die intertextuellen und historischen Bezüge betrifft. Dieses soll dem Publikum in theaterpädagogischen Workshops auf spielerische Weise vermittelt werden. Dabei macht der interaktive Ansatz die Arbeitsweise im Theater auf sinnliche Weise transparent.

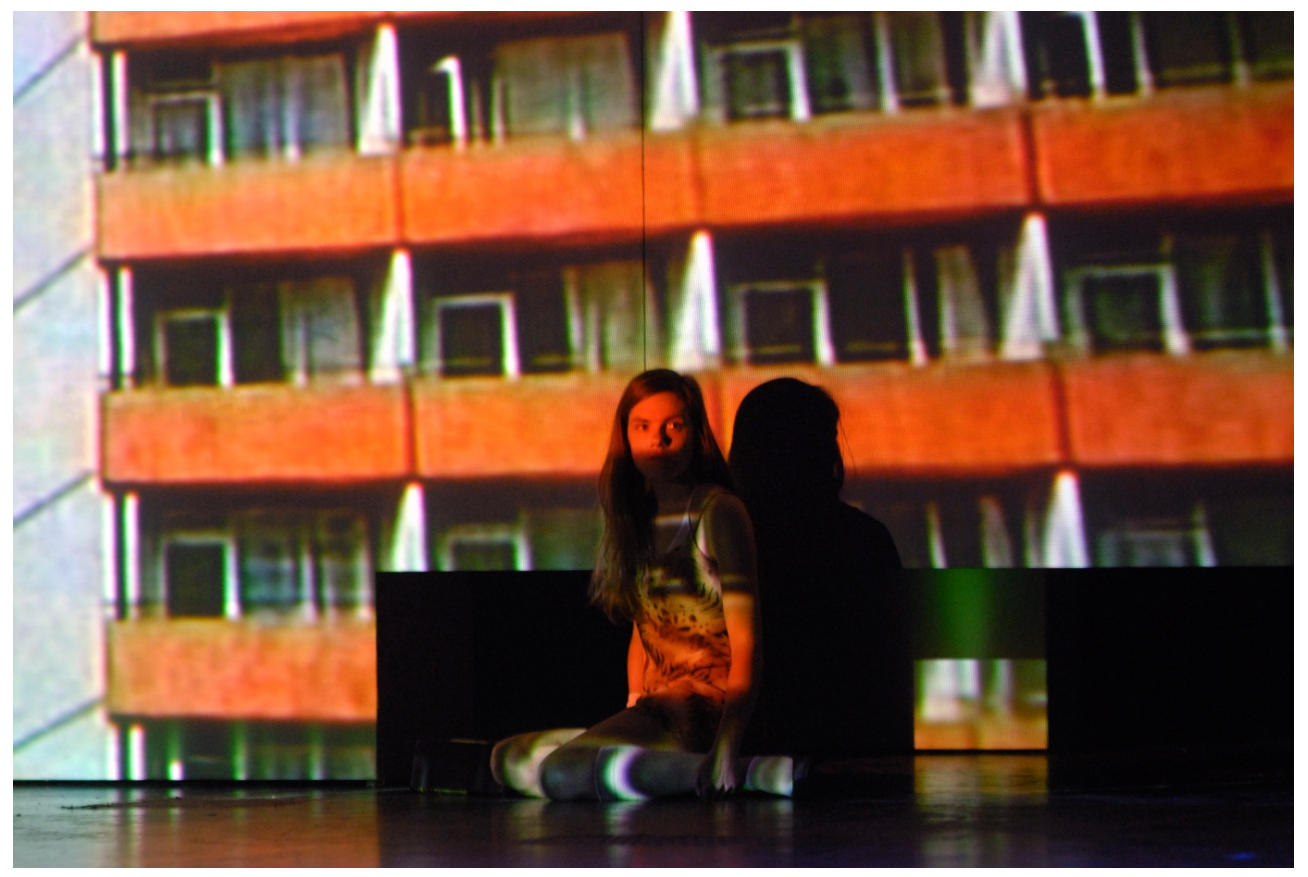

Abbildung 1: Heaven (zu Tristan)

\subsection{DER WORKSHOP}

Die Inszenierung ist eher karg in der Ausstattung. Auf einen weißen Block werden die Bilder einstürzender Neubauten projiziert. Wolfen. Eine Heimat wird geschreddert. Eine Lebenswelt verfällt vor den Augen ihrer Bewohner. 
Die Geschichten der Menschen werden erzählt als eine Tragödie auf der Folie großer Oper und großer Legenden.

Was braucht ein Zuschauer, der die Situation in Ostdeutschland nicht kennt, um sich für die Empfindungen der Figuren zu öffnen? Was brauchen die Studierenden, um die Intentionen des Autors, des Regisseurs und des Ensembles zu verstehen? Das ist die Ausgangsfrage, die ich mir als Theaterpädagogin stelle. Welche Zeichen müssen übersetzt werden? Welche Querverbindungen müssen gezogen werden?

Zuhause ist da, wo ich herkomme - Ich entschließe mich, mit einer Einfühlung in das Heimatthema zu beginnen. Jeder, der ein Zuhause hat, verbindet es mit Gefühlen und Erinnerungen. Davon zu erzählen schafft Nähe.

Übung 1 - Kennen lernen: Die Gruppe steht im Kreis. Jeder nennt seinen Namen und den Ort seiner Herkunft, verbunden mit einer Geste. Alle machen sie nach!

Das ist eine sehr simple Übung. Aber sie macht folgende fundamentale Dinge über Theater deutlich: Theater ist Nachahmung. Nachahmung ist nie perfekt, da sie durch die Gegebenheiten des eigenen Körpers und der eigenen Wahrnehmung verändert wird. Es entsteht eine Interpretation, eine subjektive Abwandlung der Vorlage.

- Ich könnte Du sein. Ich kann sehen, fühlen, verstehen, wer du bist und in deine Rolle schlüpfen. Empathie. Theater wirkt über Identifikation, über das Erkennen des Eigenen im Fremden.

- Ich könnte eine Figur auf dem Theater sein: Hiro aus Yokohama oder Ilaria aus Venedig. Ich müsste nicht all meine Privatheiten verkünden, sondern würde mich auf das Wesentliche reduzieren, auf das, was für die Geschichte wichtig ist. Theater ist abstrakt.

Übung 2 - Mein Zuhause - Eine Installation: Jeder der Gruppe schreibt ein Wort auf einen Zettel, das den Satz beendet: „Zuhause, das bedeutet für mich ...“ Auf der Bühne stehen Stühle, auf denen die Zettel dem Publikum zugewandt arrangiert werden. Jeder Teilnehmer tritt vor und erklärt sein Wort und wofür es steht. Eine Installation entsteht, die alle Zuhause zusammenfasst aus den Empfindungen aller.

Der Vorgang der Erklärung ist ein performativer. Er zeigt einen Menschen, der öffentlich von sich erzählt, der durch die Bühne vom Privatmenschen zum exemplarischen Menschen wird. Obwohl alle an der Entstehung der Installation beteiligt sind, fungieren die Zuschauenden als anteilnehmendes Publikum. Das ist eine Zuschauhaltung, die wir Theaterpädagoginnen gerne in unserem Publikum erzeugen, weg vom Konsumieren von Kunst, hin zur koproduzierenden Auseinandersetzung mit dem Kunstwerk. 
Übung 3 - Blick über eine Landschaft - Beschreiben: In der Inszenierung gibt es eine Stelle, wo Helga und Königsforst auf dem Dach ihres Hauses stehen und über die Landschaft ihrer Heimat schauen, sie zeigen ins Publikum auf einen angeblichen Wald, eine leerstehende Fabrik. In unseren Köpfen entsteht ein Panorama. Diese schauspielerische Aufgabe erteile ich den Workshopteilnehmern auch. Sie gehen einzeln vor unsere „Zuhause“-Installation auf die Bühne. Dann erzählen sie mit Worten und Gesten, wo in ihrer Heimat sie stehen und was sie von diesem Ort aus sehen können, was für Erinnerungen sie mit diesen Plätzen verbinden. Vor uns Zuschauenden entstehen Landschaften, wir sehen einem Menschen beim Denken zu, seine Gesten ordnen den imaginären Raum um ihn herum, sein Körper, sein Gesicht offenbaren Emotionen, die aus der Erinnerung emporsteigen. Die meisten sind sich der Theatralität dieses Vorganges nicht bewusst, das gedankliche Rekonstruieren des Erinnerungsbildes erfordert zu viel Konzentration. Auch die Zuschauer vergessen, dass sie Zuschauer sind. In ihren Köpfen entsteht die Vorstellung einer Landschaft, einer Kindheit.

Wir haben ein Panorama von Heimat, Zuhause und Kindheit geschaffen. Ich habe es entstehen lassen, um es im Folgenden zu zerstören, vor aller Augen, um so den Verlust, den die Figuren im Stück erleiden, deutlich zu machen. Ich kommentiere unsere Übungen jetzt erst. Ich stelle den oft warmen Erinnerungsgefühlen bewusst kalte und harte Fakten der Realität gegenüber:

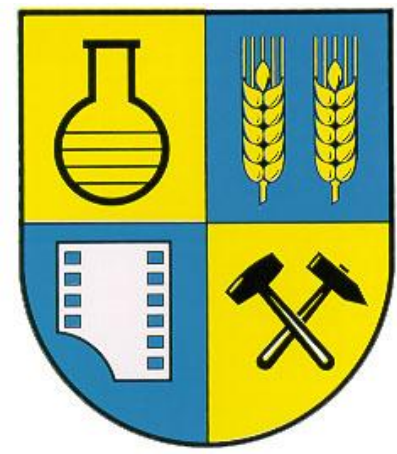

Abbildung 2: Das Wappen von Wolfen

Armin Petras ist aufgewachsen in Wolfen. Wolfen erhielt 1958 das Stadtrecht. Das Wappen von Wolfen zeigt neben Ähren auch einen Erlenmeierkolben als Symbol für chemische Industrie und einen Filmstreifen als Symbol für die Filmfabrik Wolfen (ORWO). Allein in der Filmfabrik arbeiteten 14000 Frauen. Nach der Wende wurde die Filmfabrik Wolfen geschlossen. Die Menschen verloren ihre Arbeit und die Stadt ihre Identität. 1999 hatte Wolfen noch 44000 Einwohner. 2005 waren es nur noch 25000. Seit 2007 ist Wolfen keine eigenständige Stadt mehr, sondern Stadtteil des benachbarten Bitterfeld.

Übung 4 - Debatte über sterbende Städte: Am Anfang des Stückes wird dieser Verlust der Heimat durch die Figur des jungen Architekten Anders Adlercreutz reflektiert. Aus seinem Text stammen die Sätze für die folgende Übung. 
- Sterbende Städte werden von den Bewohnern als Verlängerung ihres eigenen Körpers empfunden.

- Sie fühlen sich selber krank.

- Sie sind verunsichert und in ihrer Existenz bedroht.

- Die Tatsache, dass Gemeinden verlassen werden, ist nicht neu in der Geschichte.

- Im amerikanischen Mittelwesten, in Nordengland und im Ruhrgebiet wurden unrentable Produktionsstätten stillgelegt.

- Ganze Landstriche sind verödet.

- Das ist unangenehm für die Bevölkerung.

- Es gibt Umstrukturierungsmaßnahmen.

- Neue Technologien werden angesiedelt.

- Durch Bildungs- und Sozialoffensiven können Katastrophen verhindert werden.

- In Ostdeutschland ist die Lage heute anders.

- Milliarden von Subventionen versackten in der Bürokratie oder flossen direkt zu den Konzernen.

- Die von Staatsgeldern gebauten Infrastrukturen erwiesen sich als überdimensioniert.

- Die jüngeren gut ausgebildeten Menschen fliehen aus den Städten.

- Die älteren und die ganz jungen Menschen müssen bleiben.

- Sie leben in einer Welt, die aussieht wie früher, aber nicht mehr funktioniert.

- Alles, was von außen kommt, erleben sie als feindliche Bedrohung.

- Sie haben keine Zukunft, keine Hoffnung, keine Träume, die sie mit dem Begriff Heimat verbinden könnten.

- Wir brauchen Träume von den Orten, in denen wir leben wollen.

- Wie soll dein Haus, deine Stadt, dein Leben, dein Land in 10 oder 15 Jahren aussehen? 
Jeder Teilnehmer bekommt einen dieser Sätze. Die Teilnehmer tragen sie dann wie in einer Debatte nacheinander vor. Allerdings sollen den Sätzen Konjunktionen vorangestellt werden, die klar machen, welche Position man in Bezug auf seinen Vorredner vertritt. Schließt man sich an („Ja, genau, und“etc.) oder widerspricht man („Aber nein, ...")? Begründet man (,weil, deswegen...") oder belegt man ("zum Beispiel, genau so wie...")? etc. Durch diese Übung wird die Sprache subjektiver, dialogischer und emotionaler, die Teilnehmenden können die objektivierende Distanz zum Text aufgeben und finden so eine Haltung, die die Figur durch Körpersprache, Tonfall, Partnerbezug zusätzlich zum Textinhalt ausdrückt. Der Sachinhalt des Textes wird dadurch konnotiert, gewichtet, gerichtet, gewertet; durch Ironie und Sarkasmus kann der Inhalt sogar in sein Gegenteil verkehrt werden. Die Teilnehmenden können so selbst mit den Gestaltungsmöglichkeiten von Sprache spielen. ${ }^{2}$

Zuhause ist da, wo meine Zukunft ist? - Die Figuren im Stück haben Mühe, sich ihre Zukunft vorzustellen. Aus Mangel an Zukunft versuchen Helga und Königsforst gemeinsam aus dem Leben zu gehen. Aber auch das scheint kein Ausweg. Sie lassen sich scheiden und leben einfach weiter. Robert gebiert fortwährend neue Ideen für die Zukunft - aberwitzige oder vernünftige, visionäre oder hirnrissige. Simone ergeht sich in Traurigkeit - kann keine Zukunft denken ohne ihren Geliebten.

Zuhause ist da, wo meine Liebe ist —Katernennt sein StückHeaven (zu Tristan). Er bezieht sich dabei eher auf den Kern der Legende als auf die konkreten literarischen Vorlagen. Ihn interessiert die verhängnisvolle Verstrickung im Dreieck Tristan, Isolde, Marke, die im Zwiespalt zwischen Liebe und Loyalität keine gerechte Lösung zulässt.

Übung 5 - Tristan spielen - eine Improvisation: Die Gruppe soll sich mit der Legende von Tristan und Isolde vertraut machen. Sie soll die Geschichte selbst spielen. Drei Kleingruppen erhalten ein kurzes Dossier, das mit einfachsten Mitteln (Pappkrone, Stoffe, Holzschwert) in Szene gesetzt und den anderen vorgespielt wird. Die Tristanmotive sollen später in der Aufführung wiederentdeckt werden können.

- Anweisung für Kleingruppe 1 - Riwalin ist ein schöner junger Ritter. Er kommt an den Hof von König Marke und geht mit ihm gemeinsam in den Krieg. Dabei wird er schwer verwundet. Blanscheflur ist die Schwester des Königs Marke. Sie hat sich in Riwalin verliebt. Weil sie glaubt, dass

\footnotetext{
${ }^{2}$ Der Text selbst schlägt vor, was zu tun ist: Zukunftsvisionen zu malen, zu spielen, zu beschreiben. Wie soll mein Tag in 10 Jahren aussehen? Hier ließe sich der Workshop in eine sozialpädagogische Richtung erweitern. Aber meine Aufgabe als Theaterpädagogin an einem Theater ist die Vorbereitung eines Theaterbesuches; das heißt Wissen, Emotionen und Erfahrungen zu vermitteln, die dem Publikum die genussvolle und erkenntnisreiche Rezeption eines Theatererlebnisses ermöglichen.
} 
er sterben wird, besucht sie ihn heimlich. Die beiden lieben sich. Diese große Liebe macht ihn gesund und sie schwanger. Riwalin wird als König in seine Heimat zurückgerufen. Feinde bedrohen seinen Thron. Er nimmt Blanscheflur mit sich und heiratet sie. In der Schlacht wird er getötet. Blanscheflur erfährt von Riwalins Tod und stirbt an gebrochenem Herzen. Ihr Sohn bleibt ohne Mutter und Vater zurück. Und weil sein Leben so traurig begann, nennt man das Kind Tristan.

- Anweisung für Kleingruppe 2 - Das Waisenkind Tristan wird wie ein Königssohn an König Markes Hof aufgezogen. Aus Dankbarkeit schlägt er für ihn viele Schlachten. Einmal trifft ihn ein Giftpfeil. Ein Mädchen rettet ihm das Leben und verschwindet danach. In beiden entbrennt eine große Liebe füreinander. Tristan stellt sich einem Zweikampf. Der Preis für den Gewinner ist die Hand der Königstochter Isolde. Tristan gewinnt den Kampf. Prinzessin Isolde leibhaftig ist das Mädchen, das ihn einst gerettet hat. Aber König Marke will Isolde zur Frau. Auf der Reise zu Marke will Isolde Tristan und sich selbst vergiften. Aber ihre Dienerin hat das Gift mit einem Liebestrank vertauscht, um ihre Herrin zu retten. Jetzt lieben sich die beiden nur noch mehr.

- Anweisung für Kleingruppe 3 - Tristan ist krank vor Sehnsucht nach Isolde, die mit König Marke verheiratet ist. Auch ihr Herz schlägt seit langem für Tristan. Tristan bittet Marke, für ihn in den Krieg ziehen zu dürfen. Er kann nicht mehr in Isoldes Nähe sein. Verletzt kommt er aus der Schlacht zurück. Isolde pflegt Tristan gesund. Beide geben sich der Liebe zueinander hin. Marke entdeckt das Verhältnis und verbannt die beiden. Aber Marke liebt Isolde so sehr, dass er sie zurück holen lässt. Tristan geht ins Ausland und will nicht zurück. Er kann ohne Isolde nicht leben und nicht sterben. Er wird in einem Kampf tödlich verwundet. Isolde eilt $\mathrm{zu}$ ihm. König Marke verfolgt sie. Tristan und Isolde sterben eng umschlungen. „Isolde, meine Freude, Isolde, meine Not, du bist für mich das Leben, du bist für mich der Tod. “3

Spielende wie Zuschauende haben sich nun eine literarische Quelle angeeignet. Jetzt kommt es darauf an, deren Motive in der Inszenierung wiederzuentdecken. Der Transfer wird vorbereitet.

Der Autor Fritz Kater behauptet ${ }^{4}$ die Tragödie des Ostens. In den Grundfesten erschüttert, finden die Menschen keinen Halt mehr. Anders Adlercreutz/Tristan, der ausgezogen war eine andere/neue Heimat für sich und Simone/Isolde zu finden, kommt todkrank zurück. König Marke/Robert wischt das Blut ihrer

\footnotetext{
3 Dieses Zitat stammt aus Günter de Bruyns Nachdichtung (Frankfurt/Main, Dezember 2000) von „Tristan und Isolde“, die auf Gottfried von Strassburgs Versepos zurückgeht. In diesem Falle eignet es sich wegen seiner poetischen Dichte und Einfachheit als Textinspiration für den letzten Satz Tristans, der von den Spielenden so benutzt werden kann.

${ }^{4}$ Gegen den Vorwurf von Ostalgie und Larmoyanz, der den Ostdeutschen von Westdeutschen gerne gemacht wird, wenn sie ihre Verluste beklagen.
} 
Selbstmordversuche weg, bemüht sich verzweifelt Heimat zu geben, wo keine mehr ist.

Aber auch allen anderen Figuren ist das Glück versagt. Kater variiert das „Nicht-mit-dir-und-nicht-ohne-dich“ in dem misslingenden, gemeinsamen Selbstmord von Helga und Königsforst, in den ,kaputten' Beziehungen ihrer Tochter Sarah und in der Halt- und Hoffnungslosigkeit von Micha, den seine Mutter verlassen hat, um ihn ernähren zu können. Folgerichtig „fällt er in den Sumpf und da fressen ihn die Raben."

Simone. :

meinen bruder haben seine kumpels mit dem baseballschläger erschlagen zu ferienende warn schöner sommer nur zu heiß alle seen ham geblüht gefühl zu haben ein nichts zu sein meinem kleinsten bruder dem der noch lebt sagen sie in der schule aus dir wird nichts kann gar nichts draus werden wolfen

$\begin{array}{ll}1990 & 44000 \\ 2005 & 25000\end{array}$

macht 19000 minus plus ein micha

Der Dramatiker Kater, der sich auf Brecht, Müller und Schleef als Lehrer beruft, fügt noch mehr Schichten und Parallelen ein, die diese Grundmetapher von Heimatverlust und Entwurzelung aufgreifen. Heimat ist dabei nicht nur ein Ort, sondern auch ein gewohntes Denksystem, das erschüttert wird und zu dem es kein Zurück mehr gibt.

Königsforst. :

ICH HABE KEINEN HIMMEL MEHR ÜBER MIR

UND ICH BIN NICHT GEBOREN SONDERN

AUSGELIEFERT

MEINE PANIK IST MIT DER ZU VERGLEICHEN ALS 1630 KEPLER DIE

SONNENFLECKEN ENTDECKTE JETZT WAR klar

DASS DIE ERDE KEINE SCHEIBE MEHR SEIN KONNTE

SIE WAR

EINE KUGEL

UND MAN WÜRDE ÜBER KURZ ODER LANG VON IHR

STÜRZEN

Die Figuren selbst sind nicht nur Akteure der Geschichte. Sie reflektieren sie auch. Sie erleben ihren Untergang bei vollem Bewusstsein und wachem Verstand. Kater lässt seine Figuren von den Wissenschaftlern Marietta Blau und Viktor Hess erzählen, die einander inspirierten und deren Erforschungen der fotografischen Darstellung kosmischer Strahlung sich ergänzten und zu einer wissenschaftlichen Revolution führten. Diese brachte Viktor Hess den Nobelpreis ein, während Marietta Blau, die deutsche Jüdin, im mexikanischen 
Exil ungeehrt und unbemerkt starb. So kann es gehen. Die Umordnung der Welt fordert ihre Opfer.

Anders. :

es gibt kein anderes leben

es gibt nur das hier

und bewegung

Welche Rolle spielt regionale Verwurzelung in meinem Leben? Wie gehe ich mit den modernen Anforderungen an Flexibilität und Mobilität um? Wiegen deren Gewinne die Verluste an Heimatverbundenheit auf? Das sind die Fragen, mit denen ich die Workshop-Teilnehmenden in die Vorstellung entlasse. Im Gespräch nach der abendlichen Aufführung wird sich erweisen, wie sie sich mit dem Gesehenen in Beziehung setzen, ob und wie sie das Gesprächsangebot der Theaterleute annehmen.

Zuhause ist da, wo man um mich trauern wird - Der Dramatiker Fritz Kater erhielt für sein Stück Heaven (zu Tristan), in dem er Sterbebegleitung seiner Heimat, Trauerarbeit leistet, den Else-Lasker-Schüler-Literaturpreis.

Der Regisseur Armin Petras wurde für die Inszenierung mit dem FriedrichLuft-Kritikerpreis geehrt. Was macht dieses Stück so wichtig? Die Jury begründet ihre Entscheidung:

Fritz Kater schafft ein zeit- und grenzüberschreitendes 'Ost-Stück', das gesellschaftliche Konflikte auch als individuelle Konflikte erzählt, deutschdeutsche Gegenwart beleuchtet, ohne in Klischees zu verfallen. Armin Petras gelingt eine Inszenierung, die auch das fragmentarische, bruchstückhafte der Erzählung in sowohl packende wie auch poetische Bilder fasst und eindrucksvolle schauspielerische Leistungen provoziert, die die Zuschauer in ihren Bann ziehen.

\section{Teil ,... und nützlicher vielleicht als tausend Bücher“ - Zur Wirkung des ,Heaven-Workshops' am Maxim Gorki Theater auf internationale Studierende der Universität}

\section{Hamburg - Erinnerungs-, Reflektions- und Feedback-Splitter}

"Was machst du eigentlich mit Studierenden aus aller Welt, die an der Universität Hamburg an unterschiedlichen Fakultäten studieren und bei dir studienbegleitende DaF-Lehrveranstaltungen besuchen in einem Theaterworkshop und dazu noch in Berlin?“ Auf diese Frage antwortete ich: „Mit ihnen gemeinsam ostdeutsche Realität besser kennen lernen!“Erstaunen. „Tja, wer 17 Jahre nach der Wiedervereinigung beim Thema geografische Begrenzungen des eigenen Landes erst einmal nur auf Flensburg und Passau kommt und wem die Gegend zwischen Meiningen und Usedom immer noch unbekannter ist als Land und Leute zwischen Barcelona und Granada, sollte schleunigst mal etwas dagegen 
tun, wenn sie guten Gewissens weiter deutsche Landeskunde unterrichten möchte", füge ich noch hinzu.

Die Kooperation am Berliner Maxim Gorki Theater (MGT) mit einer gestandenen Theaterpädagogin und Schauspielerin aus Stralsund verspricht anregend, lehrreich unkompliziert zu werden. Der Umstand, dass wir in zwei unterschiedlichen Systemen aufwuchsen und ausgebildet wurden, macht das ganze Unternehmen besonders spannend. Unser großes Vertrauen in die Wirksamkeit theatraler Arbeits- und Lernformen eint uns ebenso wie unsere norddeutsche Herkunft.

Die grob orientierende Vorbereitung auf Fritz Katers Stück Heaven (zu Tristan) erfolgt für die zwölf ausländischen Studierenden aus fünf Fakultäten mit neun unterschiedlichen Sprachen, Kulturen und Herkünften im Rahmen einer zweistündigen studienbegleitenden Lehrveranstaltung Deutsch als Fremdsprache mit dem Titel „Übungen zu und mit literarischen Texten“. Dies geschieht ohne Textvorlage mit einer von der Bundeszentrale für politische Bildung herausgegebenen kleinen Landkarte der Bundesrepublik Deutschland in ihren Grenzen von 1990, Auszügen aus dem Programmheft des MGT sowie dem kurzen Essay „Rette dich Tristan! - Einmal quer durch die Republik, die es nicht mehr gibt" von Ludwig Haugk. ${ }^{5}$

Zum Einstieg führt das Sammeln von H-Wörtern zu einer poetischen Alliteration, die in konkrete studentische Fragen zu Inhalt und Problematik des Stücks münden.

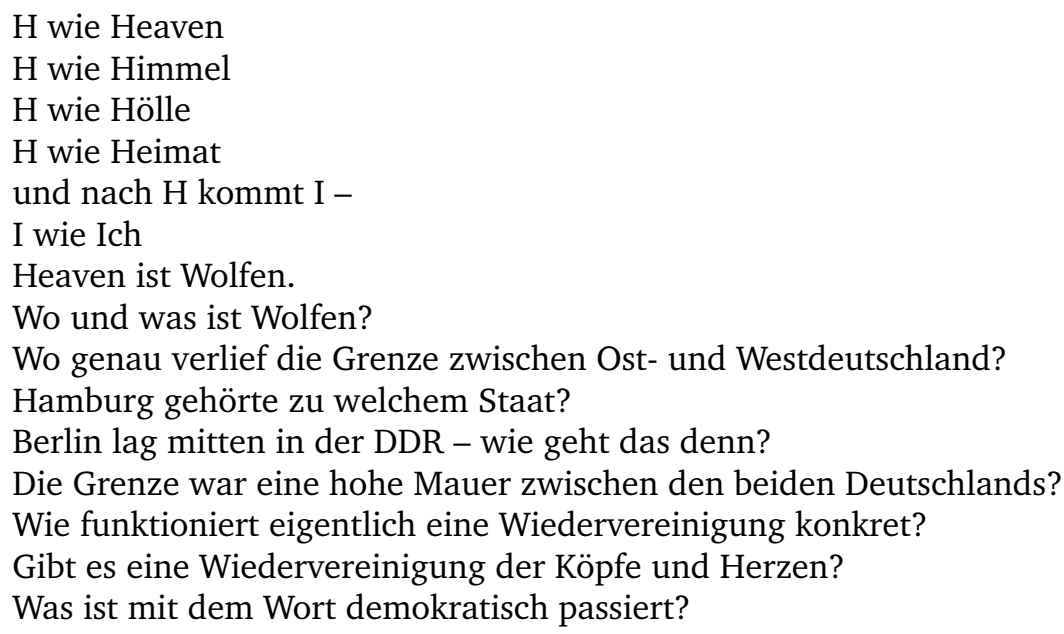

Offene und ehrliche Fragen von zwölf jungen Menschen, die Ende der 70er / Anfang der 80er Jahre zwischen Yokohama und Venedig, zwischen Leningrad und Feuerland geboren wurden. Mehr als die Hälfte war schon einmal in Berlin, Hauptstadt der Bundesrepublik Deutschland. Dieser Berlin-Besuch aber wird anders. Wir gehen zu Fuß vom neuen Hauptbahnhof aus zum MGT - zu finden in der Prachtallee Unter den Linden zwischen der Humboldt-Universität und dem

\footnotetext{
${ }^{5}$ Im Gorki-Planet, dem vierteljährlich erscheinenden Blatt, der Dramaturgie des Maxim Gorki Theaters, das den Spielplan kommentiert.
} 
Deutschen Museum im Herzen des ehemaligen Ost-Berlin, der Hauptstadt der Deutschen Demokratischen Republik. Wir bleiben immer wieder stehen. Ohne öffentliche Hinweise versuchen wir mit Hilfe eines Stadtplans zu rekonstruieren, wo denn nun die Grenze zwischen den beiden Welten in dieser einen Stadt verlief. Es ist schwer nachzuvollziehen.

Ich entscheide mich, Elemente aus dem szenischen Lernen auf offener Straße einzusetzen, um unsere Wahrnehmung und unser Verstehen von dem schwer Vorstellbaren zu unterstützen.

Wir machen einen Abstecher zum großen Gendarmenmarkt und ich rege an, eine Personeninstallation zu versuchen, indem wir markante Orte in der Stadt durch uns selbst zueinander positionieren.

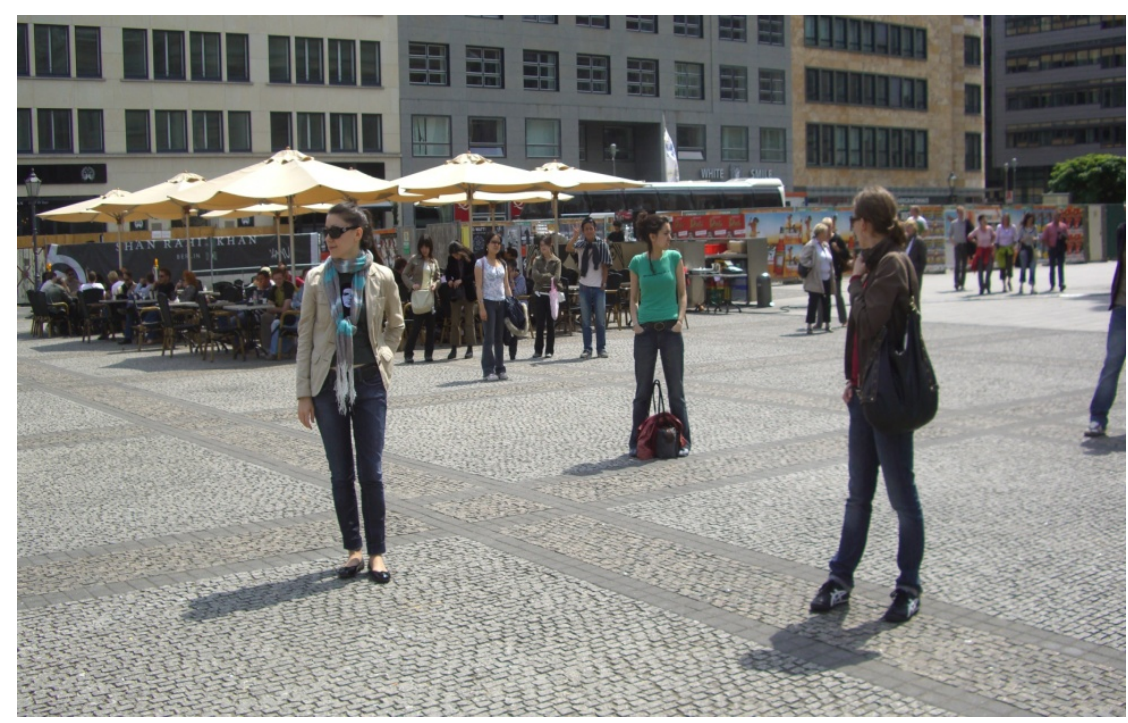

Abbildung 3: Personeninstallation

„Wenn ich der Berliner Hauptbahnhof bin und Luis das Reichstagsgebäude, wer ist dann wo das Brandenburger Tor, die Siegessäule, der Fernsehturm, der Funkturm, der Palast der Republik etc. Ein „Stadtvermesser“ erhält nach abgeschlossener Installation ein Stück Kreide und den Auftrag, stadtplanunterstützt den ungefähren Mauerverlauf auf dem Pflaster zu markieren und die noch nicht Positionierten darauf als Mauer zu installieren. Die Siegessäule reckt den Hals, sie kann die Humboldt-Universität nicht mehr sehen.

Teilung wird erlebbar. Wir vertiefen nicht weiter. Eigentlich ist das nicht unser Thema. Aber eine solche Erfahrung am eigenen Leibe, so die Annahme, ist eine gute Vorbereitung auf die bevorstehende Workshop-Arbeit und die abendliche Theateraufführung, in der viele Bezüge zum Nachwende-Deutschland (Ost) hergestellt werden.

Wir erreichen das MGT mit Verspätung. Vor allem die russischen Teilnehmerinnen sind gespannt auf die Einführung. Wie kam dieses deutsche Theater zum Namen eines ihrer ganz großen Dichter? Fragen werden beantwortet, Bühnen-Räumlichkeiten gezeigt, Theaterluft eingeatmet, und schon geht's los. 
Mitten im Foyer unter den überlebensgroßen Schauspielerfotos nehmen wir Platz. Alle sind ein bisschen aufgeregt und höchst aufmerksam.

Der Einstieg (vgl. Übung 1 in Teil I) übers Ich gelingt und ebnet alles Weitere. Niemand hatte Erfahrungen mit theatralen Arbeits- und Lernformen und es ist höchst überraschend, wie schnell sich alle öffnen, nach außen wie nach innen.

Die DIN-A4-Blatt-1-Wort-Installation zur Frage „Was ist Heimat für dich?" bringt berührende Wortschöpfungen durch ungewöhnlichen Einsatz von Suffixen oder Präfixen zu Tage: wie z.B. warmherzlich oder Uneinsamkeit .

Eine mich erstaunende Spontaneität lässt die Studierenden schüchtern bis resolut - aber angstfrei und flüssig - ihre Kommentierungen darbieten. Beim mündlich vorgetragenen inneren Blick über eine Landschaft - eine Landschaft oder einen Ort der Erinnerung - lernen sich alle noch einmal ganz anders kennen. Die Wehmut über das Vergangene oder das Verlorene oder das im Moment Nicht-Erreichbare lässt sie etwas näher zusammenrücken. Man merkt ihnen an, dass das Thema Zuhause / Heimat sie berührt hat, es anfängt sie zu fesseln und aufnahmebereiter macht für das postostdeutsche Besondere, das für sie Neue und Fremde.

Die sich anschließende Übung (vgl. Übung 4 in Teil I) steigt unmittelbar in das Stück ein, indem die Workshop-TeilnehmerInnen mit einzelnen Sätzen zur Problematik der sterbenden Städte aus der Heaven-Theaterfassung ausgestattet werden. Beim Vortragen ergänzen sie die Sätze durch Satz verbindende Elemente, treten so in direkten Dialog miteinander über das verödende, sterbende Wolfen im Osten Deutschlands und kommentieren später im Auswertungsgespräch mit ihren herkunftsbezogenen Erfahrungen und Informationen entvölkerte Städte im Norden Japans (Toya/Hokkaido), verwahrloste Ansiedlungen im Westen Sibiriens und zerstörten Lebensraum im Süden Chiles, dem äußersten Süden der Welt.

Neben dem Umstand, dass es sich um eine jedem DaF-Unterricht alle Ehre machende, zum Sprechen animierende und rhetorische Kompetenz fördernde Übung handelt, scheinen die Studierenden mit allen Sinnen im dem Workshop Raum gebenden Theaterfoyer angekommen zu sein. Die kurze Debatte wird - mimisch und gestisch unterstützt - mit Leidenschaft geführt, befreit von der üblichen nüchternen Unterrichtssituation, und lässt die Sätze lockerer und überzeugender zugleich kommen.

Die Mini-Inszenierungen zu Tristan und Isolde mit den ewigen Menschheitsthemen Liebe, Leidenschaft, Treue, Verrat lassen sie vollends heimisch werden in den recht eigenwilligen Foyer-Gegebenheiten: vor der Garderobe, neben den Säulen, an Spiegeln, vor Flügeltüren - überall wird mit Bravour geschmachtet, geliebt, gestritten, gekämpft, gestorben.

Ich war der König von Irland und die Dienerin ${ }^{6}$

Bewegend bewegt durch die eben durchlebten Kurz-Dramen ist es ein Leichtes,

\footnotetext{
${ }^{6}$ Kursiv geschriebene Zitate sind den schriftlichen Auswertungen der ausländischen Studierenden entnommen.
} 
bei der nächsten und letzten Übung mit Rollenkarten in Figuren zu schlüpfen, die wir am Abend auf der Bühne erleben werden.

Etwas abgekämpft, müde - und den Hunger ganz vergessend - scheinen die Studierenden sich schon der Inszenierung zugehörig zu fühlen, ein wenig wie ein Schattenensemble.

Ich war doch gerade noch Simone. Nun sehe ich da oben eine andere als Simone und vor ihr den dringlichen Königsforst. Ich bin sie und sie ist ich. Simone oben und unten.

Nur noch wenig Zeit bis zum Vorstellungsbeginn, langsam füllt sich das Foyer, wo doch eben noch Tristan-Tufan seine Isolde-Ilaria beweinte. Wir nehmen unsere Plätze ein und fühlen uns gut vorbereitet. Was uns dann erwartete, konnten wir alle nicht ahnen. Eine nicht gerade leicht zu verdauende Kost bietet uns Armin Petras mit Textfülle, Erzählsträngen und Inszenierungskapriolen. Der Bauch rebelliert und uns schwirrt der Kopf, aber wir sind ja gut vorbereitet und verlieren selbigen deshalb auch nicht, sondern werden neugierig.

Ich brauche das Stück, ich will es ganz lesen und verstehen. Und dann wieder kommen und noch einmal oder auch zweimal ansehen.

Brennende Fragen werden nach der Vorstellung direkt besprochen. In der dafür stets bereiten Theaterkantine natürlich. Bärbel Jogschies und noch ein paar Theaterleute stehen zur Verfügung und stellen Wein, Wasser, Bier sowie die legendären Gorki-Frikadellen bereit. Ein mitgebrachter Hamburger Journalist gesellt sich zur Runde. Theatertage haben irgendwie mehr als 24 Stunden. Es geht auf Mitternacht zu. Macht nichts, die Berliner Verkehrsbetriebe fahren rund um die Uhr.

Nach vielerlei Nachfragen zum eben erlebten Bühnenereignis geht es um die gelebte Realität heute, vor zehn, vor zwanzig und vor dreißig Jahren im geteilten und ungeteilten doch noch geteilten Land: wie war das mit dem Alltag, mit der Ausbildung, mit der Freiheit, mit Mann und Frau und, und, und... ? Beim Antworten kriegen sich Ost und West etwas in die Wolle, und die aus Hamburg zum Workshop mitgekommenen Studenten aus aller Welt verfolgen fasziniert bis irritiert den kleinen Schlagabtausch.

Ich dachte, ich weiß viel über die beiden Deutschlands, aber letzte Nacht habe ich gemerkt, dass ich lange bleiben muss, um zu verstehen, was hier mit den Menschen passiert ist und noch passiert - in Berlin scheint man mehr Antworten zu finden als in Hamburg.

Aufgewühlt und nachdenklich, erschöpft, aber nicht müde, schreiben sie und ich am folgenden Tag im Zug zurück nach Hamburg auf, was wir denken und fühlen über die vergangenen 36 Stunden in Berlin.

Ich notiere: 
Landes- und Kulturkunde:

Gegenwartsliteratur/Gegenwartstheater:

Sprech- und Handlungskompetenz:

Ästhetische Bildung:

theatrale Kreativität: erlaufen und (dar)gestellt,

eingestiegen und angespielt

ganzheitlich trainiert

interkulturell praktiziert

geweckt und freigesetzt

Fazit: begeisterte Erschöpfung. Im Sommersemester wieder ins MGT zu B.J.

Es folgt ein kollektiver poetischer Versuch von 12 Personen aus 9 Ländern:

tortur-kultur-licht

Eine emotionale Erfahrung!

vierecke-stühle-bordeaux

Ich war der König von Irland und die Dienerin.

folie-raum-projekt

Wenn ein Boden defekt ist, dann wächst nichts Gutes daraus.

spiel-geschichten-Menschen

Zeit und Menschen ändern sich - und die Menschen?

wir-heimat-ich

In kurzer Zeit waren in meinem Kopf so viele Bilder.

geräusche-gerüche-erinnerungen

arbeit-team-drama

Ganz neue Erfahrung und ganz große Motivation: hat mir soviel Spaß

gemacht.

wein-suche-spannend

ich freue mich so, dass ich alle unsere Leute besser kennen lernen konnte.

unterricht-zeit-achtsamkeit

Und wenn Gefühle dabei sind, dann ist es sonderbar -

und nützlicher vielleicht als tausend Bücher...

\section{Nachtrag}

Susana mailte vor einigen Monaten aus Lugano: „Ich werde meine Magisterarbeit zu Fritz Kater schreiben, „Heaven“ habe ich mir schon besorgt und auch gelesen...", und in einer zweiten Mitteilung vor kurzem: „Ich habe einen Praktikumsplatz am MGT bekommen! Wahnsinn!“ 